\title{
Blood Folate Concentrations Analyzed by Microbiological Assay and Chemiluminescent Immunoassay Methods
}

\author{
Mio NAKAZATO ${ }^{1}$, Takahiro MAEDA ${ }^{1}$, Kosuke EmurA ${ }^{1}$, Mayu MAEDA ${ }^{1}$ \\ and Tsunenobu TAMURA ${ }^{2}$ \\ ${ }^{1}$ Nagasaki University Graduate School of Biomedical Sciences, Goto, Nagasaki 853-8691, Japan \\ ${ }^{2}$ University of Alabama at Birmingham, Birmingham, Alabama, USA
}

(Received September 6, 2011)

\begin{abstract}
Summary A limited number of studies have been available to compare blood folate concentrations by the microbiological assay (MA) method with those using the chemiluminescent immunoassay (CLIA) method. We compared folate concentrations measured by Lactobacillus rhamnosus MA with those measured by CLIA (Access Immunoassay Systems) in human plasma/serum and erythrocytes pairs $(n=35)$. The mean plasma folate by MA was significantly higher than that by CLIA $(p<0.0001)$, whereas the mean erythrocyte folate by MA was significantly lower than that by the CLIA method $(p<0.001)$. Plasma folate by MA significantly correlated with serum folate by CLIA $(r=0.85, p<0.001)$. Similarly, the correlation between erythrocyte folate measured by MA and CLIA methods was significant $(r=0.87, p<0.001)$. We conclude that folate concentrations obtained by CLIA are different from those obtained by MA, suggesting that it is undesirable for inter-laboratory comparisons when folate values are obtained by different methods. Although we evaluated only one CLIA method, we recommend careful evaluation of folate assay by each CLIA method before the use in clinical and research settings.
\end{abstract}

Key Words folate, plasma, erythrocyte, microbiological assay, chemiluminescent immunoassay

Blood folate concentrations have most commonly been used as an indicator of nutritional status for folate. Plasma/serum folate concentrations are influenced by recent folate intakes, whereas erythrocyte folate concentrations are believed to reflect a long-term intake of folate. For decades, blood folate analysis has been performed by microbiological assay (MA) using Lactobacillus rhamnosus (formerly L. casei, ATCC 7469), which is considered the gold standard of the assay (1). However, the inter-laboratory discrepancies among the values by the method have been reported $(2,3)$. Due to laborintensive and time-consuming issues, researchers have avoided using the MA method, although the microplate-reader assay has made the analytical procedure less laborious (1). In clinical laboratories, methods other than the MA, such as radio-binding (RBM) and automated chemiluminescent immunoassay (CLIA), have been used since the $1970 \mathrm{~s}$ and $1990 \mathrm{~s}$, respectively. A liquid-chromatography/mass-spectrometric method has been introduced mainly for research $(4,5)$, although it remains unclear whether the method will be widely used for clinical settings.

Due to the radioactivity involvement, the use of the RBM has faded away. Furthermore, this method gives falsely high values for folates other than pteroylglutamic acid (folic acid) or monoglutamyl 5-methyltetrahydrofolate (5- $\mathrm{CH}_{3}-\mathrm{H}_{4}$ folate), including 10 -formyltet-

E-mail: mio@msb.biglobe.ne.jp rahydrofolate (10-HCO-H${ }_{4}$ folate) and folate polyglutamates (6). Therefore, the CLIA method has become most popular in clinical laboratories and certain research settings. However, no systematic studies have been made to validate the CLIA method under research settings. There have been studies to compare folate values among various CLIA methods or to compare the values by the CLIA method with those obtained by MA or other assay methods $(2,7-10)$. We compared folate values in human plasma/serum and erythrocytes measured by L. rhamnosus MA with those by a CLIA method.

\section{Materials and Methods}

This study was reviewed and approved by the committee of Nagasaki University based on the Declaration of Helsinki (project registration: 090528160). Fasting blood was obtained using evacuated tubes with or without EDTA (Terumo, Tokyo, Japan) for plasma/serum and erythrocyte folate assays from 35 healthy Japanese adults (27 non-pregnant females and 8 males) who were non-smokers without history of serious disease. Their mean age was 73.1 ( \pm 9.2 , SD; range 53-92) y old with the mean weight of $51.5( \pm 9.9) \mathrm{kg}$. Samples were obtained in June 2009. Portions of whole blood for erythrocyte folate measurement were mixed with 9 volumes of ascorbic acid solution $(56.8 \mathrm{mmol} / \mathrm{L})$ or $20 \mathrm{vol}$ umes of ascorbic acid solution $(8.5 \mathrm{mmol} / \mathrm{L}$ : supplied by the manufacturer) for the whole-blood folate measure- 
ment by the MA and CLIA method (Access Immunoassay System, Beckman Coulter, Tokyo, Japan), respectively. According to the package insert (2008, Access Folate, WB A33383, Beckman Coulter), whole blood lysate was incubated at room temperature for $90 \mathrm{~min}$ for hydrolysis of polyglutamyl folate by endogenous plasma folylpoly- $\gamma$-glutamate carboxypeptidase II (folate conjugase) (1). Plasma was mixed with an equal volume of $56.8 \mathrm{mmol} / \mathrm{L}$ ascorbic acid solution and kept frozen at $-30^{\circ} \mathrm{C}$. Ascorbic acid was added to these samples to protect against folate degradation, because the time lapse between blood drawing and the assay was unknown. Serum samples were placed at $-30^{\circ} \mathrm{C}$ immediately after separation and shipped on dry ice to a commercial laboratory (SRL Inc., Tokyo, Japan), who requested serum for folate analysis by CLIA. Both serum and whole blood folate assay was performed in July 2009. After 4 mo of blood drawing, both whole-blood lysate and plasma were sent on dry ice to Birmingham (USA), where the MA assay was done in October 2009. MA was performed using [6RS]-5-formyltetrahydrofolate (5-HCO-H 4 folate, calcium salt, Sigma, St. Louis, MO, USA) as a standard. The biological activity of the standard was considered $50 \%$ after the optical density analysis of the concentration $(1,11)$. Before the assay, whole-blood lysate was incubated for $1 \mathrm{~h}$ at $37^{\circ} \mathrm{C}$ to hydrolyze erythrocyte folate polyglutamates by plasma folate conjugase. Our inter-assay coefficient of variation (CV) for MA was $\sim 6 \%$ using 2 pooled human plasma samples. Our values of Standard Reference Material 1955 (National Institute of Standards and Technology, Gaithersburg, MD, USA) were within all 3 specified levels (12). According to the package insert obtained in 2007, the inter-assay CVs ranged $1.8-4.8 \%$ and $2.3-$ $4.2 \%$ for serum and whole blood, respectively. Erythrocyte folate value was calculated using a formula: [whole-blood value - plasma value $\times(1-$ hematocrit $(\%)$ / $100)] \div$ hematocrit/100. All values are presented as mean $( \pm S D)$. For the correlation between the two methods, linear-regression analysis and the Bland-Altman bias plot were used. For the differences between the two methods, the paired Student $t$-test was used. All statistical analyses were performed using PASW Statistics $18^{\circledR}$ software (IBM Japan, Tokyo, Japan). A $p$ less than 0.05 was considered significant.

\section{Results and Discussion}

The mean plasma folate concentration measured by MA was significantly higher than the mean serum folate by CLIA and the difference was almost two-fold $(p<0.0001$, Table 1$)$. We do not believe that the difference was due to the difference in the sample preparation (plasma versus serum), since it has been well established that there is no significant difference in folate values between plasma (with EDTA or heparin) and serum samples (13). Our findings are similar to the data from Ihara et al. (9) who reported that serum folate values by CLIA (Access) were lower than those by MA. Furthermore, the correlation coefficient of serum folate values by the two methods according to Ihara et al. (9)
Table 1. Folate concentrations (nmol/L, mean \pm SD) analyzed by Lactobacillus rhamnosus microbiological assay (MA) and chemiluminescent immunoassay (CLIA) in 35 human blood samples.

\begin{tabular}{lccc}
\hline & MA & CLIA & $p^{*}$ \\
\hline Plasma (with EDTA) & $25.2 \pm 14.5$ & & \\
Serum & & $13.0 \pm 5.2$ & $<0.0001$ \\
Erythrocyte & $466 \pm 231$ & $546 \pm 220$ & $<0.001$ \\
\hline
\end{tabular}

* Significance for the difference between the two methods.

was 0.82 , which was close to ours $(r=0.85)$.

In contrast to the plasma and serum relation, the mean whole blood folate concentration (203 \pm 101 $\mathrm{nmol} / \mathrm{L})$ measured by MA was significantly lower than that $(226 \pm 88 \mathrm{nmol} / \mathrm{L})$ of whole blood folate concentrations by CLIA $(p=0.006)$. Moreover, the mean erythrocyte folate concentration calculated on the basis on plasma and whole blood folate concentrations (MA) with hematocrit values was significantly lower than those calculated using values from CLIA $(p<0.001$, Table 1).

Although the mechanism for the differences in the two methods remains to be elucidated, a few possible reasons can be offered. Firstly, there is no information given by the manufacturer ("Access A14208" from Beckman Coulter on October 6, 2009) as to what folate standard was used for this assay and how the concentrations of their calibrators were determined; according to Ihara et al. (9), it is folic acid. This is critical information, as suggested by Owen and Roberts (7) who were the first to compare serum and whole blood folate concentrations determined by CLIA kits produced by several companies with those by the RBM (BioRad, CA, USA). They reported that serum or whole blood folate measured by CLIA varies widely among products and do not always agree with those by RBM. Although the exact mechanism for such discrepancies is unknown, they reported that folate standards may not be uniform among manufacturers (7). Secondly, it is possible that the anti-folate binding protein (mouse) and/or milk folate-binding protein (bovine) used for CLIA have a limited ability to bind to certain folates. For example, the package insert (2008, Access Folate, WB A33383, Beckman Coulter) indicates that cross-reactivity with folinic acid ( $5-\mathrm{HCO}-\mathrm{H}_{4}$ folate) was less than $1.1 \%$ of the added $100 \mathrm{ng} / \mathrm{mL}$ of this folate. Thirdly, 10-HCO$\mathrm{H}_{4}$ folate has been identified in erythrocytes of individuals with the thermolabile variant $(677 \mathrm{C}>\mathrm{T})$ of gene coding 5,10-methylene-tetrahydrofolate reductase (14). Therefore, certain folates might not have been fully detected by this particular CLIA method.

A specificity problem may exist in the CLIA method as noted by Clifford et al. (5). They reported that using CLIA, erythrocyte folate concentrations in pregnant women were higher than those in non-pregnant women, although no such difference was found by MA. 

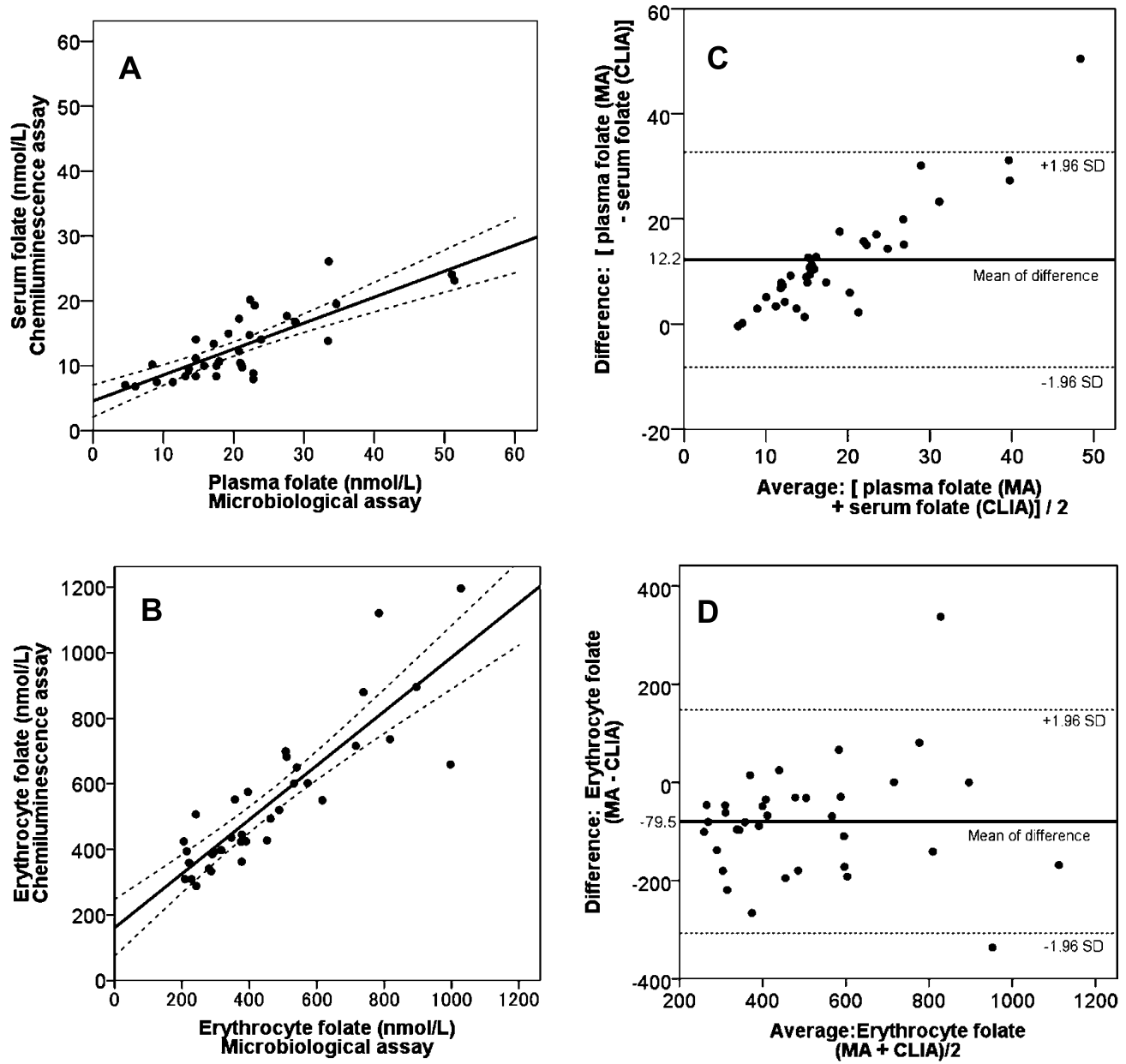

Fig. 1. Relationship of folate values in plasma and serum measured by microbiological assay and chemiluminescence immunoassay (CLIA) methods. (A) The correlation coefficient between plasma and serum folate concentrations was 0.85 $(p<0.001)$. The equation of the regression line was "serum folate concentrations $(C L I A)=0.30 \times$ plasma folate concentrations (microbiological assay)+5.3." (B) The correlation coefficient of erythrocyte folate concentrations by two methods was $0.87(p<0.001)$, and the equation of the regression line was "erythrocyte folate concentrations (CLIA)= $0.83 \times$ erythrocyte folate concentrations (microbiological assay)+161." For both A and B, broken lines represent 95\% confidence intervals. (C) The correlation coefficient of Bland-Altman bias plots for plasma versus serum concentrations from the two methods was $0.92(p<0.001)$, and the equation was "differences (plasma-serum) $=1.01$ (95\% CI $0.85-$ 1.16) $\times$ average $-6.96(95 \% \mathrm{CI}-10.27--3.65)$. (D) The correlation coefficient of Bland-Altman bias for erythrocyte values from the two methods was $0.10(p=0.28)$ with an equation of "differences between the two methods=0.06 (95\% CI $-0.13-0.24) \times$ average $-107(95 \% \mathrm{CI}-210--4.0)$.

Their findings suggest a possible lack of folate specificity in CLIA (possible presence of pregnancy-related interfering substance in samples). Because there were no pregnant women in our study, we were unable to evaluate such a potential difference.

Plasma folate values obtained by MA significantly correlated with serum values by CLIA $(r=0.85$, $p<0.001$, Fig. 1A). However, the slope of the correlation was only 0.30 (95\% confidence interval [CI] $0.24-$ 0.37 ) with the intercept of 5.3 (95\% CI 3.4-7.3), indicating marked differences between the two methods. Similarly, the correlation between erythrocyte folate concentrations measured by the MA and CLIA methods was significant $(r=0.87, p<0.001)$ with the slope of 0.85 (95\% CI $0.66-0.99)$ and the intercept of 161 (95\% CI 75-248, Fig. 1B). Clifford et al. (5) reported the correlation $(r=0.61, p<0.0001)$ in erythrocyte folate concentrations determined by MA and CLIA (Bayer
Diagnostics ADVIA Centaur). They also found that erythrocyte folate values from CLIA were $\sim 13 \%$ higher than those from MA, which is close to the difference in erythrocyte folate from two methods in our study (about $17 \%$ higher for CLIA).

We constructed Bland-Altman bias plots for serum versus plasma and for the sets of erythrocyte folate from the two methods. A significant correlation between serum and plasma values $(r=0.92, p<0.0001$, Fig. 1C) indicated the presence of a proportional bias. In contrast, no significant correlation was found in erythrocyte values from the two methods $(r=0.10, p=0.55$, Fig. 1D) with a significant difference between the two methods $(p<0.001)$, indicating the presence of a fixed bias. The linear-regression analysis indicated that the correlation between plasma and erythrocyte folate values from MA was significantly positive $(r=0.74$, $p<0.001)$ and was similar between serum and erythro- 
cyte folate from CLIA $(r=0.68, p<0.001)$.

The weaknesses of our study include: 1) the number of subjects was small with a narrow age range, and it remains to be determined if our findings apply to other populations; 2) the MA method was compared to only one of several CLIA methods commercially available. It is unknown whether our data can be extrapolated to other CLIA; and 3) there were differences in samples (serum versus plasma) and their handling (amount of ascorbic acid added and dilution of whole samples). It would be desirable to minimize such controllable variables in the future studies.

In conclusion, our data indicate that folate values obtained by CLIA are different from those by MA. Further studies are warranted to explore the mechanism(s) of the differences between the values obtained by these two methods.

\section{Acknowledgments}

Financial support for this work was provided by a research grant for Scientific Research from the Japan Society (21770267).

\section{REFERENCES}

1) Tamura T. 1990. Microbiological assay of folates. In: Folic Acid Metabolism in Health and Disease (Picciano MF, Stokstad ELR, Gregory JF III, eds), p 121-137. Wiley-Liss, New York.

2) Gunter EW, Bowman BA, Caudill SP, Twite DB, Adams MJ, Sampson EJ. 1996. Results of an international round robin for serum and whole-blood folate. Clin Chem 42: 1689-1694.

3) Pfeiffer CM, Mindy Zhang M, Lacher DA, Molloy A, Tamura T, Yetley EA, Picciano MF, Clifford L, Johnson CL. 2011. Comparison of serum and red blood cell folate microbiologic assays for national population surveys. $J$ Nutr 141: 1402-1409.

4) Pfeiffer CM, Fazili Z, McCoy L, Zhang M, Gunter EW. 2004. Determination of folate vitamers in human serum by stable-isotope-dilution tandem mass spectrometry and comparison with radioassay and microbiologic assay. Clin Chem 50: 423-432.

5) Clifford AJ, Noceti EM, Block-Joy A, Block T, Block G.
2005. Erythrocyte folate and its response to folic acid supplementation is assay dependent in women. J Nutr 135: 137-143.

6) Shane B, Tamura T, Stokstad ELR. 1980. Folate assay: a comparison of radioassay and microbiological methods. Clin Chim Acta 100: 13-19.

7) Owen WE, Roberts WL. 2003. Comparison of five automated serum and whole blood folate assays. Am J Clin Pathol 120: 121-126.

8) Bock JL, Endres DB, Elin RJ, Wang E, Rosenzweig B, Klee GG. 2005. Comparison of fresh frozen serum to traditional proficiency testing material in a College of American Pathologist survey for ferritin, folate and vitamin $\mathrm{B}_{12}$. Arch Pathol Lab Med 129: 323-327.

9) Ihara H, Hashizume N, Totani M, Inage H, Kimura S, Nagamura Y, Sudo K, Aoki Y, Saeki H, Sagawa N, Kamioka K, Shimizu K, Watanabe R, Watanabe M, Hirayama K, Nakamori M, Takenami K, Yoshida M, Kawasaki Y, Ogiwara T, Kawai T, Watanabe T. 2008. Traditional reference values for serum vitamin $\mathrm{B}_{12}$ and folate are not applicable to automated serum vitamin $\mathrm{B}_{12}$ and folate assays: comparison of value from three automated serum vitamin $\mathrm{B}_{12}$ and folate assays. J Anal Bio-Sci 31 : 291-298.

10) Pfeiffer CM, Fazili Z, Zhang M. 2010. Folate analytical methodology. In: Folate in Health and Disease (Bailey LB, ed), p 517-574. CRC Press, Boca Raton.

11) Tamura T, Freeberg LE, Cornwell PE. 1990. Inhibition by EDTA of growth of Lactobacillus casei in the folate microbiological assay and its reversal by added manganese or iron. Clin Chem 36: 1993.

12) Satterfield MB, Sniegoski LT, Sharpless KE, Welch MJ, Hornikova A, Zhang N-F, Pfeiffer CM, Fazili Z, Zhang M, Nelson BC. 2006. Development of a new standard reference material: SRM 1955 (homocysteine and folate in human serum). Anal Bioanal Chem 385: 612-622.

13) O'Broin JD, Temperley IJ, Scott JM. 1980. Erythrocyte, plasma, and serum folate: specimen stability before microbiological assay. Clin Chem 26: 522-524.

14) Bagley PJ, Selhub J. 1998. A common mutation in the methylenetetrahydrofolate reductase gene is associated with an accumulation of formylated tetrahydrofolate in red blood cells. Proc Natl Acad Sci USA 95: 1321713220. 